\title{
EXPERIENCIAS DE APEGO EN MADRES DE NIÑOS CON RETRASO MADURATIVO: ANÁLISIS DE CONTENIDO
}

\section{Attachment experiences in mothers of children with developmental delay: content analysis}

\author{
Maritza García Toro \\ Universidad de Salamanca \\ magato@usal.es \\ M. ${ }^{a}$ Cruz SÁNCHez Gómez \\ Universidad de Salamanca
}

Recepción: 16 de abril de 2018

Aceptación definitiva: 20 de noviembre de 2019

Resumen: El objetivo de este estudio es elaborar un perfil de percepciones, emociones y opiniones en torno a experiencias de apego de un grupo de madres de niños con retraso madurativo, a través del análisis de contenido de un foro virtual. A partir de los datos se extraen tres dimensiones de análisis: sistema de conductas del niño, sistema de cuidados y crianza mediada por profesionales. Los resultados permiten detectar como temas recurrentes de preocupación en las madres los siguientes: incertidumbre por el diagnóstico; dificultades para interpretar la conducta de sus hijos y responder consecuentemente; búsqueda de intimidad afectiva, y predominio del aprendizaje guiado durante las primeras etapas del diagnóstico. Se concluye que la categoría retraso madurativo, al ser provisional, dificulta el proceso de aceptación, lo cual impacta negativamente en el sistema de cuidados materno. Al respecto, la terminología, actitudes y prácticas de los profesionales de apoyo parecen jugar un papel importante. El deseo de lograr intimidad afectiva, las emociones positivas y un adecuado acompañamiento profesional serían factores protectores para establecer vínculos de apego más favorables entre las figuras parentales y sus hijos.

PALABRAS ClAve: retraso madurativo; apego; investigación cualitativa; foro virtual.

Aвstract: The aim of this study is to develop a profile of attachment experiences through the analysis of content shared in a virtual forum by a group of mothers 
who have children with developmental delay. Three categories were extracted from the data: behavioural system, care system, and professional parenting assistance. The results allowed the identification of the mothers' emotional states associated with the uncertainty about diagnosis, difficulty interpreting their children behaviour, and responding consequently, seeking emotional intimacy, and prevalence of guided learning during the early stages of diagnosis. It is concluded that the diagnosis "developmental delay", being provisional, makes the acceptance process difficult, which impacts the maternal care system negatively. Terminology, attitudes, and practices of support professionals seem to play an important role. The desire for emotional intimacy, positive emotions, and appropriate professional support would be protective factors to establish a more favourable attachment between parents and children.

KEY WORDs: developmental delay; attachment; qualitative research; virtual forum.

\section{Introducción}

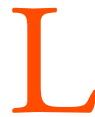

a Teoría del Apego ha ocupado un lugar central en la comprensión del desarrollo psicoafectivo del infante y las relaciones adultas. Bowlby $(1969,1973$, 1980) estableció que el apego es cualquier comportamiento orientado a alcanzar o conservar proximidad con respecto a otro individuo diferenciado y preferido, subrayando que dicho vínculo permite construir representaciones mentales sobre la figura de apego, sobre sí mismo y la relación con esta. Mientras tanto, Ainsworth, Blehar, Waters y Wall (1978) pusieron el énfasis en la disponibilidad del adulto como cuidador, a la hora de conceptualizar el apego, y establecieron la importancia de la sensibilidad parental, definiéndola como la habilidad de los padres (o cuidadores) para identificar e interpretar con precisión los signos de apego de sus hijos, además de la capacidad y disposición para responder oportuna y adecuadamente a estas señales. A partir de allí, definieron tres tipos de apego dependiendo del estilo de los cuidadores y la respuesta del niño a sus cuidados (apego seguro, evitativo y resistenteambivalente). Bowlby $(1969,1973,1980)$ y Ainsworth et al. (1978) coincidían en que el vínculo de apego brinda estabilidad y tranquilidad cuando se está seguro de la disponibilidad de la figura de apego y de la capacidad de esta para ayudar.

Existe un importante volumen de investigaciones en torno al apego en distintas discapacidades y alteraciones del desarrollo como los trastornos del espectro del autismo (Rutgers, 2007; van Ijzendoorn et al., 2007), los trastornos del lenguaje y la discapacidad intelectual, incluyendo el síndrome de Down (Dyches, Smith, Korth, Roper y Mandleco, 2012; Fiamenghi, Vedovato, Meirelles y Shimoda, 2010; Howe, 2006; Schuengel, Clasien de Schipper, Sterkenburg y Kef, 2013), pero hay menos estudios que se enfoquen directamente en el retraso madurativo como categoría diagnóstica provisional y el impacto que esto puede tener en la disposición emocional de las figuras parentales.

Cuando se habla de retraso madurativo se hace referencia a la demora o alteración en la secuencia normal de adquisición de los hitos del desarrollo, durante los primeros años de vida (Czornyj, 2004; Narbona y Schlumberger, 2008). Esta categoría abarca 
una variedad de signos que pueden suponer una afectación de la maduración neurológica, cuya duración e intensidad dependen de factores individuales preexistentes, así como de factores ambientales, tales como la deprivación sociocultural, la hipoestimulación, la malnutrición, entre otros (Campos-Castelló, 2013). De modo que este retraso implica un diagnóstico provisional, pues en algunos casos el retraso madurativo es signo de una discapacidad o de dificultades de aprendizaje, pero, en otros casos, existirá la posibilidad de un acercamiento a la "normalidad". Es por esto que dicha categoría se reserva para los niños menores de seis años cuyos signos clínicos no permiten emitir un dictamen fiable, cuando las valoraciones no son concluyentes o la edad y condiciones del niño dificultan el uso de pruebas estandarizadas (Czornyj, 2004).

Cuando un niño es diagnosticado con retraso madurativo, las figuras parentales deben hacer ajustes a las expectativas acerca de su hijo y emprender el camino hacia la resolución de los sentimientos asociados al diagnóstico, pero también deben efectuar una reorganización de los patrones de interacción parental, como la comunicación, la transmisión de normas, el cuidado y la protección. Investigaciones previas han señalado que las condiciones médicas en la infancia y las discapacidades del desarrollo, en interacción con las emociones maternas, pueden afectar los patrones de interacción entre la madre y el niño, lo que a su vez puede influir en la seguridad del vínculo madre-hijo (LaMont, 2011). Se ha encontrado que las alteraciones del apego en niños con discapacidades del desarrollo podrían explicarse por sus dificultades para desarrollar habilidades cognitivas, como la permanencia del objeto y la capacidad de detectar medios para conseguir un fin (Pérez-Salas y Santelices, 2009). Al respecto, van Ijzendoorn et al. (2007) señalan que los niños con discapacidad intelectual responden menos ante los estímulos externos y sus interacciones con las figuras de apego son más escasas o menos eficaces que las de niños sin discapacidad. También se ha indicado que las conductas de apego de estos niños son menos predecibles y sus señales emocionales son más inciertas y confusas, lo cual podría sobrepasar la capacidad de los padres para interpretar y responder sensiblemente a sus necesidades (Howe, 2006; Schuengel, Kef, Damen y Worm, 2010; Schuengel et al., 2013), lo que contribuiría a una menor seguridad y una mayor desorganización del apego en el infante.

Un estudio llevado a cabo por Lewallen y Neece (2015) encontró que los niños con retraso madurativo pueden tener dificultades para diferenciar los comportamientos apropiados en función de los contextos sociales, lo cual genera frustración y estrés en los padres; intervenir el estrés parental podría ayudar a mejorar la consistencia en la transmisión de normas y el desarrollo de competencias sociales en los niños.

También se ha hallado que la capacidad de respuesta del cuidador puede afectarse por el grado de resolución del diagnóstico; así, a mayor resolución, mayor capacidad para ajustar el comportamiento parental a las necesidades del niño (Feniger-Schaal y Oppenheim, 2013). Un fallo en la aceptación del diagnóstico puede hacer que los cuidadores estén enfocados en sentimientos de confusión, culpa, ansiedad, enojo o rechazo (Chandramuki, Venkata y Vranda, 2012), lo que produce un agotamiento de los recursos emocionales para manejar las demandas que implica criar un niño con discapacidad (Pérez-Salas y Santelices, 2009; van Ijzendoorn et al., 2007). La aceptación parental no solo tiene implicaciones para el bienestar de los cuidadores, sino 
también para el desarrollo del infante (Janssen, Schuengel y Stolk, 2002), pues un fallo en la resolución del diagnóstico puede interferir con la capacidad de los cuidadores para responder de manera apropiada a las necesidades y demandas emocionales del niño (Feniger-Schaal y Oppenheim, 2013; Oppenheim et al., 2007; Teague, Newman, Tonge y Gray, 2018). Por otra parte, las actitudes de sobreprotección limitarían el desarrollo de habilidades y la asunción de responsabilidades en el niño, al restringir la exploración y el aprendizaje a partir del error.

No obstante, también hay estudios que resaltan que una alteración en el desarrollo en sí misma no es incompatible con el desarrollo de un patrón de apego seguro. Los investigadores coinciden en que el tipo o la severidad de la discapacidad del niño no son condiciones per se que originen apegos inseguros, sino que es la interacción entre los factores de vulnerabilidad del niño y de su cuidador lo que afecta la sensibilidad, la comunicación y la seguridad del apego (Dyches et al., 2012; Howe, 2006; van Ijzendoorn et al., 2007).

Por otro lado, se ha establecido que los profesionales que establecen una comunicación empática y sensible, brindando información clara y realista -pero esperanzadora- sobre el diagnóstico logran atender mejor las necesidades de las familias de un niño con una discapacidad del desarrollo (Fletcher, 2016). Sin embargo, se ha encontrado que algunos profesionales de atención temprana cuentan con conocimientos sobre desarrollo cognitivo, motor y del lenguaje, pero sus habilidades o conocimientos con respecto al apego en discapacidad no son suficientes para abordar apropiadamente este proceso (Alexander, Frederico y Long, 2018). Diversos autores subrayan que las acciones coordinadas y la comunicación efectiva entre los distintos agentes de la educación (familia y educadores), así como los profesionales de atención temprana, psicólogos, trabajadores sociales, entre otros, ayudan a una mejor percepción de autoeficacia parental y al establecimiento de vínculos de apego favorables entre el niño y sus cuidadores (Floyd y Dago, 2014; Martínez y Calet, 2015). Asimismo, es necesaria la vinculación de la familia extensa en los programas de intervención y la oportunidad de conocer a otras familias en la misma situación para disminuir la sensación de aislamiento y mejorar la satisfacción parental (Fletcher, 2016; Hsieh, Hsieh y Lee, 2016).

El objetivo de la presente investigación es elaborar un perfil de las percepciones, emociones y opiniones en torno a experiencias de apego de un grupo de madres de niños con retraso madurativo que frecuentan un foro virtual. Concretamente se busca identificar qué conductas del niño ponen de relieve, qué componentes de la interacción resultan problemáticos y cuáles son sus respuestas a esto; así mismo, cómo describen su experiencia emocional. Se espera que los hallazgos permitan orientar las intervenciones profesionales en favor de la construcción del vínculo de apego.

\section{Método}

Se utilizó un método de carácter mixto CUAL_cuan (cualitativo y cuantitativo) (Delgado, 2014). La aproximación cualitativa nos permite comprender la perspectiva de las participantes acerca del fenómeno, profundizando en sus experiencias, opiniones 
y sentimientos (Sánchez, Delgado y Santos, 2012). Como análisis auxiliares que permitieran representar mejor la realidad analizada, nos apoyamos en el rigor formal de la metodología cuantitativa (Delgado, 2014; Rodríguez y Gómez, 2010). Dentro de la variedad de modelos mixtos, el diseño utilizado responde al "de conversión o transformación”. En primer lugar, se realizó un análisis de carácter interpretativo para obtener el perfil de experiencias de apego de las madres y, posteriormente, se recurrió a la cuantificación del dato cualitativo para determinar las dimensiones más representativas descritas en los comentarios analizados, como se expondrá más adelante (Obtención y gestión de datos).

La técnica empleada fue la observación participante, modo interactivo de recogida de información que requiere la implicación del observador en los fenómenos observados y permite acercarse a una parte del escenario subjetivo desde el espacio (en este caso virtual) donde las personas interactúan (Rodríguez y Gómez, 2010). Dentro de esta técnica, se adoptó el rol de participación activa, donde el investigador está inmerso en el grupo observado; de este modo, el investigador está registrado como usuario del foro, interactúa con los participantes y es admitido como parte del grupo, lo que permite que disminuyan las inhibiciones y las respuestas de deseabilidad social.

\subsection{Población y muestra}

Se realizó un muestreo razonado o intencional (Creswell, 2013; Hernández, Fernández y Baptista, 2014; Tójar, 2006), en el que no se busca una representatividad cuantitativa sino discursiva, pues el objetivo es perfilar distintas perspectivas de la misma situación y recopilar diversidad de opiniones, sentimientos y experiencias en torno al tema. Este tipo de muestreo implica que los sujetos no son elegidos por las leyes del azar, sino de alguna forma intencional, controlada.

Se recurrió al foro de discusión, debido a que permite acceder a un número amplio de familias entre las cuales, posteriormente, se delimitó la muestra. Además, porque se trata de una herramienta versátil, donde afloran discursos textuales sumativos e interactivos de carácter social y comunitario y permite la elaboración de discursos más reflexivos al no estar mediados por la instantaneidad (Arriazu, 2007) y el anonimato ejerce un efecto desinhibidor sobre los usuarios, disminuyendo también las respuestas basadas en deseabilidad social.

La selección del foro se realizó luego de un rastreo en páginas web de asociaciones de familias de niños con discapacidad, redes sociales y motores de búsqueda. Se tuvieron en cuenta los siguientes criterios: que el tema central fueran las discapacidades del desarrollo, que estuviera principalmente dirigido a las familias y que se tratara de una comunidad virtual activa en la actualidad.

El foro elegido se centra en diversidad funcional, fue fundado en 2005 por la madre de un niño con retraso madurativo y está compuesto por cuatro subforos, cada uno subdividido en temáticas: a) Presentación, espacio para compartir información personal y darse a conocer a los demás miembros de la comunidad virtual; b) Diagnósticos, donde se encuentran las temáticas relacionadas con las distintas condiciones diagnósticas (retraso madurativo, parálisis cerebral, síndromes, epilepsia, entre otros); 
c) Objetivos y logros, destinado a los avances de cada niño; d) El diván, dedicado a situaciones no relacionadas con el diagnóstico de los niños. Para el presente trabajo, se tomaron mensajes del subforo Diagnósticos y la temática Retraso madurativo. Es importante señalar que, aunque el foro cuenta con 10.257 usuarios registrados, muchos de ellos se encuentran inactivos o se trata de usuarios pasivos, es decir, que visitan el foro para consultar información, pero no participan de las discusiones.

Para la selección de los mensajes no se establecieron criterios previos relacionados con la edad, la nacionalidad o el género de los participantes; en su lugar, se examinaron textos que contuvieran narraciones detalladas y representativas de la situación bajo estudio y expresaran diversidad de opiniones y experiencias para explicar mejor el fenómeno. A través del muestreo teórico, se seleccionan casos a estudiar según su potencial para ayudar a refinar o expandir los conceptos y las teorías ya desarrollados. Lo importante no es el número de casos, sino la potencialidad de cada uno para ayudar al investigador a desarrollar una mayor comprensión teórica sobre el área que está estudiando. Por esta razón, se consideró suficiente la muestra cuando se alcanzó la saturación discursiva, de acuerdo con los siguientes criterios: los textos no aportaban nuevos datos o nuevos temas y se contaba con información rica, profunda y completa para cada categoría de análisis (Sánchez et al., 2012). Para disminuir la posibilidad de que los usuarios no fueran reales, se hizo un rastreo de temas en un plazo de dos años (1 de abril de 2013 - 30 de abril de 2015) y se identificaron participantes activos que brindaran en su perfil información personal básica. Se excluyeron los textos de madres de niños con diagnóstico de trastorno del espectro del autismo -TEA- o trastorno específico del lenguaje-TEL-, igualmente, se descartaron los mensajes que no hacían alusión al apego o al desarrollo emocional.

Finalmente, la muestra estuvo compuesta del discurso de 17 usuarias de nacionalidad española que se identificaron como madres de niños con retraso madurativo, para un total de 348 mensajes analizados. No se cuenta con opiniones de padres, pues había pocos hombres registrados y no participaron en las discusiones referidas al apego. El rango de edad de las madres es de 27-44 años $(M=38,9 ; D t=3,8)$; la edad de los niños está entre los 2-7 años $(M=3,4 ; D t=1,4)$, en su mayoría varones $(82 \%)$.

\subsection{Obtención y gestión de datos}

Después de la lectura inicial de los mensajes del foro se crearon nuevos temas de discusión, con el fin de ampliar información sobre tópicos relevantes al tema abordado (ver Tabla 1). Luego, se seleccionaron los temas y mensajes relacionados con el desarrollo emocional y el apego. Una vez que el dato empírico (en este caso, texto) fue identificado, se crearon "categorías" a través de un proceso de codificación del dato. Es un proceso intensivo donde se analiza el material empírico línea por línea y párrafo por párrafo. En principio, las categorías fueron de dos tipos: aquellas que se encontraron directamente porque fueron manifestadas por las madres, categorías "in-vivo", y aquellas que el equipo investigador construyó a partir del material y la bibliografía, categorías "in- vitro". 


\section{EXPERIENCIAS DE APEGO EN MADRES DE NIÑOS CON RETRASO MADURATIVO: ANÁLISIS DE CONTENIDO \\ MARITZA GARCÍA TORO Y M. ${ }^{a}$ CRUZ SÁNCHEZ GÓMEZ}

El proceso se desarrolló de la siguiente forma: en primer lugar, se revisaron los datos (transcripciones), se detectaron los conceptos o ideas clave y los comentarios recurrentes para extraer las categorías centrales, anotando dimensiones (subcategorías) y datos que se pudieran incluir en ellas. Posteriormente, se recogieron los textos que proporcionaban información acerca de las categorías centrales con la vista puesta en la diversidad de dimensiones bajo las categorías (subcategorías) y se inspeccionaron los datos y categorías a través del denominado en investigación cualitativa "proceso mental", que consiste en dar vueltas a las categorías en todas las formas posibles y utilizar la relación causa-consecuencia en el análisis de las mismas.

De esta forma, se compararon continuamente los datos recientemente codificados en una categoría con los datos anteriormente codificados en esa categoría. El trabajo con la codificación continuó hasta que se ha alcanzado el nivel de "saturación teórica”, es decir, cuando nuevos análisis no contribuyeron a descubrir una nueva categoría. A partir de estas categorías y de la relación entre ellas, se construyó el mapa conceptual de categorías y la relación entre ellas.

A continuación, se realizaron codificaciones intra- e interinvestigadores. En la primera cada investigador, de forma independiente, revisó su codificación en distintos tiempos. Posteriormente, y fusionando las codificaciones individuales en un archivo

\begin{tabular}{|c|c|}
\hline TÓPICO & PREGUNTAS O PROPUESTAS DE DISCUSIÓN \\
\hline $\begin{array}{l}\text { Relación con profesionales } \\
\text { y prioridades en evaluacio- } \\
\text { nes y tratamientos }\end{array}$ & $\begin{array}{l}\text { En las evaluaciones y tratamientos, ¿se ha tenido en cuenta la } \\
\text { dimensión emocional de manera explícita? } \\
\text { ¿Cómo se aborda esta dimensión? }\end{array}$ \\
\hline $\begin{array}{l}\text { Proceso de aceptación del } \\
\text { diagnóstico }\end{array}$ & $\begin{array}{l}\text { ¿Qué pasa cuando el diagnóstico no es claro? ¿Cómo afecta a la } \\
\text { espera de diagnóstico la “elaboración del duelo"? (sentimientos } \\
\text { y pensamientos). } \\
\text { ¿Cómo afecta a la relación con los niños? } \\
\text { Desde su experiencia, ¿habría alguna forma de elaborar el duelo } \\
\text { aún sin un diagnóstico claro? ¿Tiene la aceptación (duelo) "nive- } \\
\text { les” o matices? }\end{array}$ \\
\hline Comunicación & $\begin{array}{l}\text { Interpretar necesidades de los hijos cuando tienen alteraciones } \\
\text { del lenguaje. }\end{array}$ \\
\hline Conductas de apego & $\begin{array}{l}\text { ¿Quién suele tomar la iniciativa en el contacto físico u otras con- } \\
\text { ductas que reflejan apego? }\end{array}$ \\
\hline Autonomía vs. protección & $\begin{array}{l}\text { ¿Cómo intentan encontrar el límite entre darles autonomía y } \\
\text { protegerlos? }\end{array}$ \\
\hline Dimensión emocional & $\begin{array}{l}\text { Hablar de las características (en la dimensión emocional) del niño } \\
\text { independientemente de su diagnóstico. }\end{array}$ \\
\hline Disponibilidad parental & $\begin{array}{l}\text { [Con respecto a] ajustes “inconscientes" o por "instinto" que se } \\
\text { hacen para estar disponible y reaccionar adecuadamente a los pe- } \\
\text { ques }(\ldots) \text { ¿. Creen que otras personas de la familia también logran } \\
\text { esa conexión? }\end{array}$ \\
\hline
\end{tabular}


conjunto del programa Nvivo, se efectuó una segunda codificación para aquellas unidades textuales en las que no había una alta concondancia de codificación. Esto se hizo hasta obtener un porcentaje de acuerdo del $100 \%(x=1)$.

Con ayuda del programa informático NVIVO 10 se consultaron las diez palabras más frecuentes, eliminando las “palabras vacías”, es decir, aquellas que carecen de significatividad dentro del discurso; esto se comprobó a través de árboles de palabras (ver Figuras 3 y 4) para ver el significante en contexto. Posteriormente, para analizar la agrupación entre categorías se solicitó al programa elaborar análisis de conglomerados según similitud de codificación con coeficiente de Pearson, y para la visualización de resultados se eligió un dendrograma horizontal, un mapa de conexiones para apreciar las correspondencias entre categorías.

\subsection{Análisis y control de la calidad del dato cualitativo}

Se estructuraron los datos en categorías, en función de lo que consigna la literatura científica sobre el apego y se identificaron las categorías emergentes. Para garantizar el rigor metodológico, la pertinencia del marco teórico y de la codificación de información se consultaron expertos en metodología cualitativa, apego y discapacidad. La confirmabilidad de la información y la interpretación se ve reflejada mediante la aportación de árboles de palabras con segmentos literales del discurso de las madres.

\subsection{Categorías a estudiar}

La Figura 1 representa las percepciones, sentimientos y opiniones de las madres acerca de la construcción del vínculo de apego con sus hijos y otros aspectos relevantes de la dimensión emocional en la díada madre-hijo. Está compuesta por tres grandes categorías: sistema de conductas del niño, sistema de cuidados y crianza mediada por profesionales. De cada categoría se desprenden, a su vez, los temas más recurrentes relacionados con el apego.

\subsubsection{Sistema de conductas del niño}

El sistema de conductas de apego está orientado a lograr y mantener la proximidad, el contacto y la comunicación con la figura de apego. A través de dichas conductas se forma el vínculo y luego operan como mediadoras en la relación (López y Ortiz, 2001), manteniéndola dentro de ciertos límites de distancia y accesibilidad. Aquellas que las madres del foro ponen de relieve, ya sea porque están presentes o ausentes, son contacto físico, contacto visual, exploración y formas de comunicación. También aluden a otros componentes de este sistema, tales como quién es la figura de apego, episodios de ansiedad de separación y el reencuentro tras la separación, expresión y manejo emocional de los niños, además de formas de la socialización y el juego. 


\subsubsection{Sistema de cuidados}

El cuidador como promotor del apego también tiene una predisposición a conductas que permiten la construcción del vínculo. Existen algunas características que favorecen que este sistema opere de acuerdo a su función: a) sincronía afectiva, marcada por periodos de acción-pausa, que se reflejan en los mensajes que transmiten las expresiones faciales y los tiempos de interpretar-responder; b) desformalización, que hace alusión a un código de comunicación no formal o poco convencional que se establece entre la figura de apego y el niño; y c) multifuncionalidad de la interacción, es decir, que la relación con la figura de apego sirva a distintos propósitos, entre ellos, la protección y el cuidado, la expresión de la afectividad, el juego y el placer (López y Ortiz, 2001).

Los componentes que se identifican en el relato de las madres son el proceso de aceptación del diagnóstico (nombrado como duelo), incondicionalidad, intimidad, flexibilidad, autonomía, control, protección, aprendizaje guiado y sensibilidad parental, dentro de la cual se distinguen tres tipos de contenido: experiencia emocional, interpretación y respuesta a las conductas del niño.

\subsubsection{Crianza mediada por profesionales}

Se trata de una categoría emergente y está relacionada con la necesidad de contar con apoyo profesional para interpretar y responder a las conductas de sus hijos, así

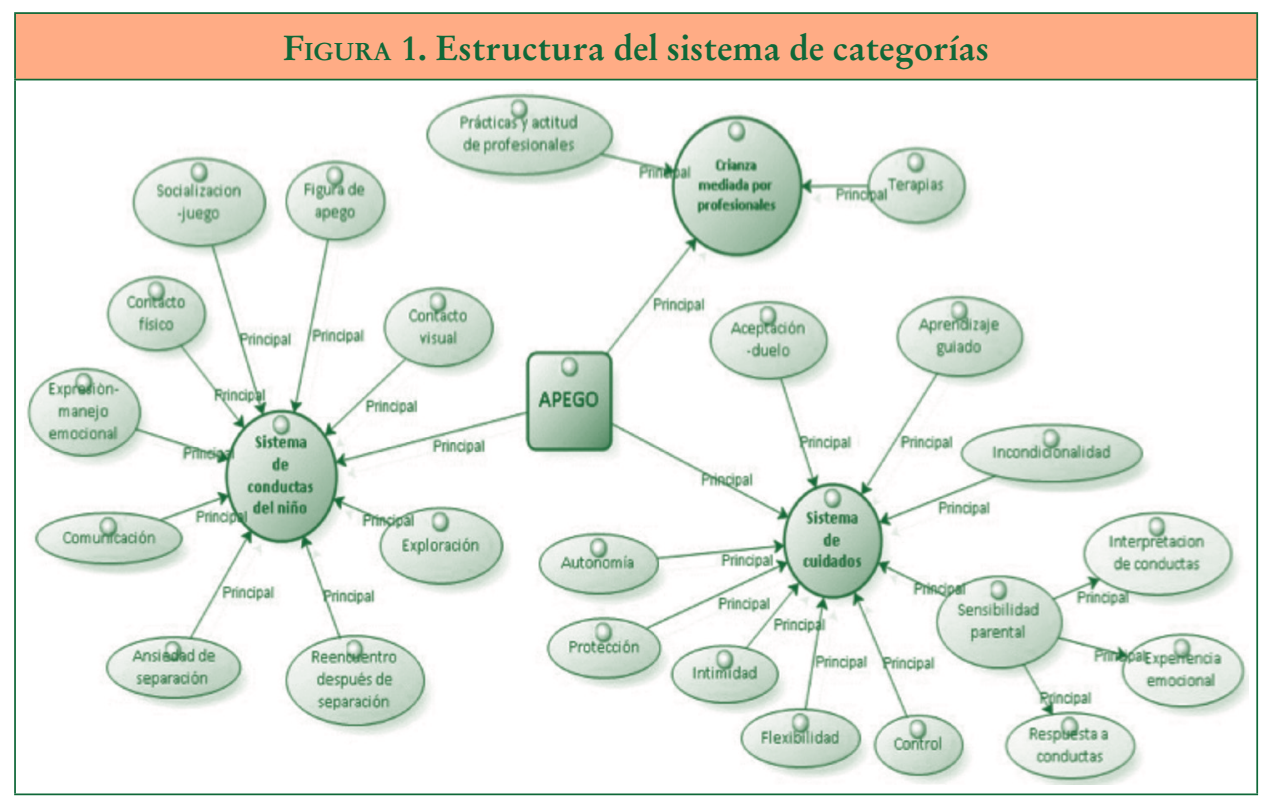

Ediciones Universidad de Salamanca / CC BY-NC-ND

Siglo Cero, vol. 51 (1), 2020, enero-marzo, pp. 89-109 
como para promover el desarrollo de habilidades. En esta categoría se identifican dos temáticas: Terapias, donde se alude a las intervenciones a las que acuden con sus hijos; y Actitud y prácticas profesionales, en la que relatan las interacciones con los profesionales durante el proceso de evaluación, diagnóstico e intervención (tanto en los programas de Atención Temprana (AT) como los equipos de apoyo a la inclusión escolar).

\section{Resultados}

La Figura 2 ilustra las palabras más recurrentes en el discurso de las madres, donde el tamaño de la palabra representa la frecuencia. Por su parte, los árboles de palabras ofrecen un panorama del contexto en el que son empleadas.

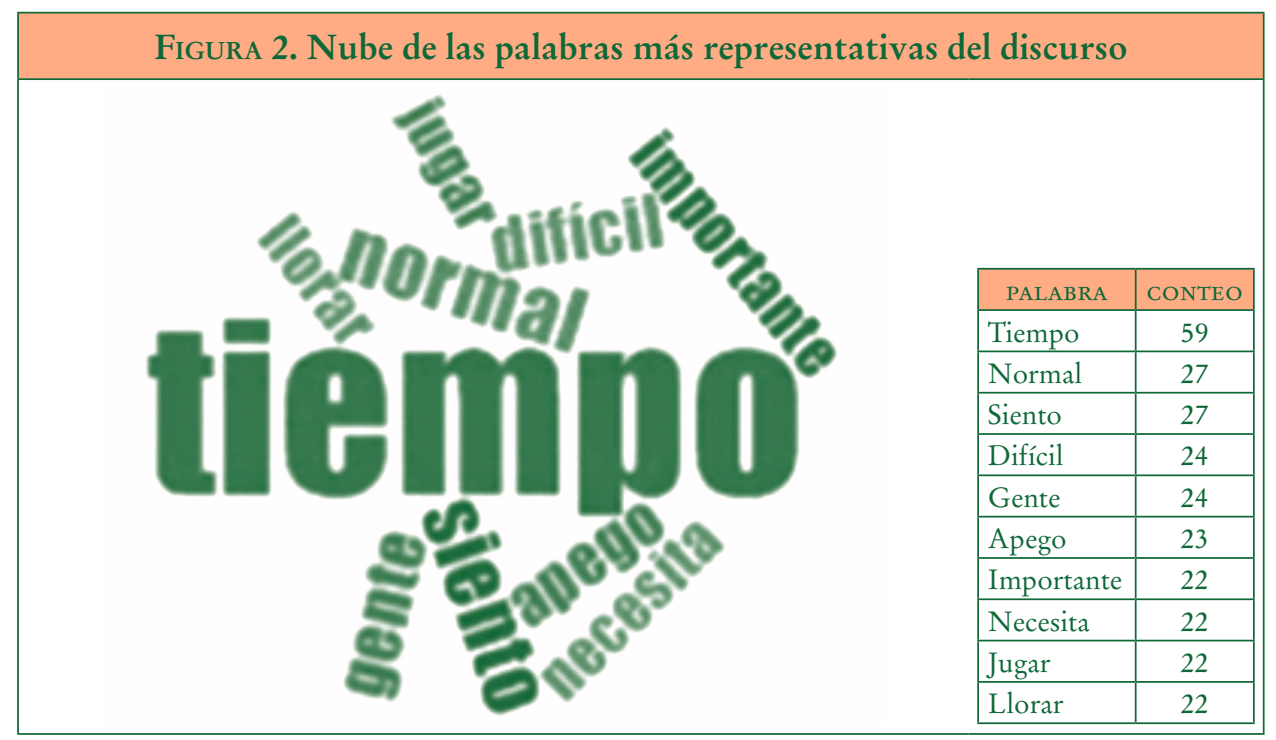

Fuente: NVIVO 10.

En primer lugar, se identifica que el diagnóstico de "retraso madurativo", al ser una categoría provisional, inserta la dimensión temporal como un aspecto relevante, pues las familias deben esperar por un diagnóstico definitivo o aguardar la remisión de las dificultades de sus hijos; espera que, de acuerdo con las madres del foro, puede extenderse varios años, interfiriendo con el proceso de aceptación. En segundo lugar, el paso del tiempo está ligado a los avances, retrocesos o "estancamiento" del desarrollo de habilidades y destrezas de los pequeños. Se resalta la falta de tiempo para el desempeño de actividades cotidianas, debido a la introducción de nuevas rutinas relacionadas con las valoraciones y terapias. Y finalmente, aunque nombrada con menor frecuencia, aparece una preocupación por el futuro de sus hijos. 


\section{EXPERIENCIAS DE APEGO EN MADRES DE NIÑOS CON RETRASO MADURATIVO: ANÁLISIS DE CONTENIDO \\ MARITZA GARCÍA TORO Y M. ${ }^{a}$ CRUZ SÁNCHEZ GÓMEZ}

La segunda palabra que se hace presente con regularidad es normal y está ligada tanto al reajuste de las expectativas como a las rutinas familiares. Nuevamente, se vislumbra una relación con el proceso de aceptación de la condición del niño.

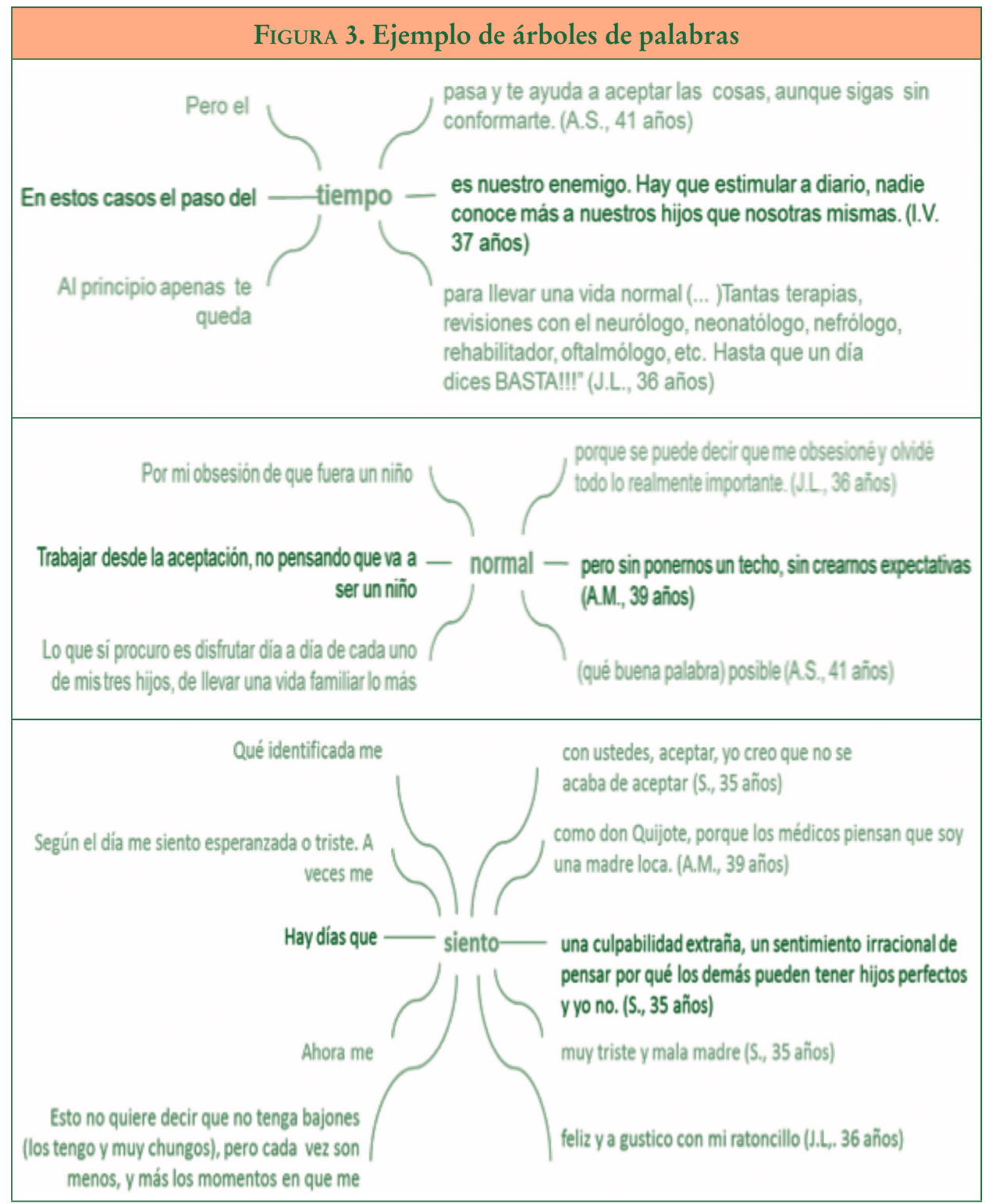

Edición a partir de resultados de NVIVO 10.

Ediciones Universidad de Salamanca / CC BY-NC-ND

Siglo Cero, vol. 51 (1), 2020, enero-marzo, pp. 89-109 


\section{EXPERIENCIAS DE APEGO EN MADRES DE NIÑOS CON RETRASO MADURATIVO: ANÁLISIS DE CONTENIDO \\ MARITZA GARCÍA TORO Y M. ${ }^{\text {a }}$ CRUZ SÁNCHEZ GÓMEZ}

Otra palabra frecuente es siento, que permite visualizar las emociones y sentimientos más comunes en las participantes: identificación con el relato de otras madres, culpa, tristeza, alegría, esperanza y desesperanza, emociones que son fluctuantes debido a la incertidumbre que trae consigo el diagnóstico.

Les siguen la palabra difícil y gente. La primera aparece en el contexto de la aceptación del diagnóstico, así como en lo concerniente a interpretar y responder a las conductas del niño. Aquí surgen narrativas acerca de la tendencia a interpretar la mayoría de las características del niño a partir de su diagnóstico, tanto por parte de los profesionales como de las familias. Mientras que la palabra gente recoge, por un lado, el malestar por las opiniones e intromisión de personas ajenas a la crianza y, por otro lado, la reacción o actitud de los niños ante los extraños.

A continuación, la palabra más recurrente es apego y aparece primordialmente como un criterio diagnóstico, en especial en niños con características confusas donde se ha sospechado TEA, sin embargo, al mismo tiempo las participantes perciben que la dimensión emocional se tiene en cuenta para el diagnóstico, mas no suele ser foco de intervención en los programas de AT. También se nombra como un elemento que causa expectativa o frustración, pues es uno de los componentes más relevantes de la experiencia materna.

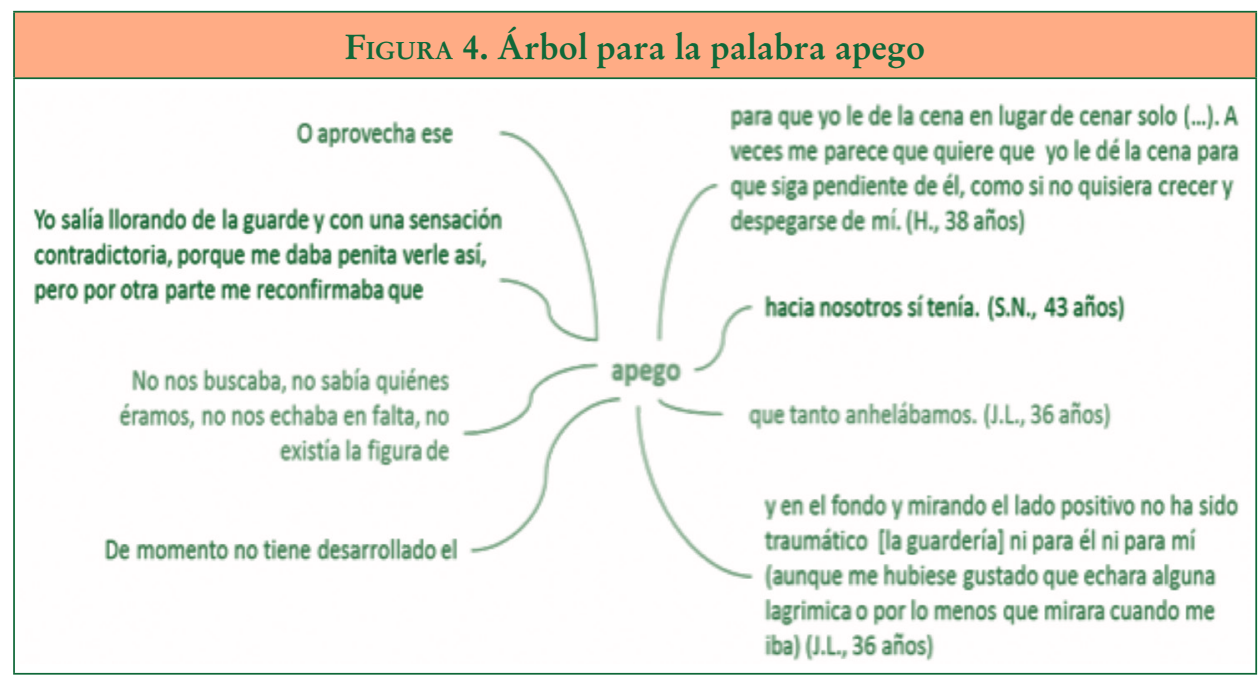

Edición a partir de resultados de NVIVO 10.

Las siguientes palabras apuntan a lo que consideran importante dentro de su rol materno y las necesidades que detectan en sus hijos. En primer lugar, es importante "aceptar a su hijo como es", y en este punto los relatos muestran, en algunas madres, el camino desde la aceptación de las dificultades hacia la valoración del niño; y, en segundo lugar, "luchar” por la superación o disminución de las dificultades, la adquisición de logros en el lenguaje y atender las necesidades de su hijo. 


\section{EXPERIENCIAS DE APEGO EN MADRES DE NIÑOS CON RETRASO MADURATIVO: ANÁLISIS DE CONTENIDO \\ MARITZA GARCÍA TORO Y M. ${ }^{a}$ CRUZ SÁNCHEZ GÓMEZ}

En esta misma línea, las necesidades del niño más nombradas son atención, protección, afecto y apoyo. Aparece nuevamente la incertidumbre por interpretar correctamente dichas necesidades y se entrevé un intento de las madres por estar disponibles pese a su estado emocional. A continuación, figuran las palabras jugar y llorar: la primera para describir el juego de sus hijos, aspecto de interés para las madres, y la segunda para nombrar tanto el estado emocional parental como una manifestación de apego del niño. Respecto a esto último, las madres evalúan el apego de sus hijos por la presencia o ausencia de llanto durante los episodios de separación.

\subsection{Relaciones entre categorías}

Tal como se puede rastrear en los árboles de palabras, algunos significados se entrelazan y conciernen a distintas categorías, por lo que se hará un análisis de las relaciones entre estas a través de los dendrogramas del análisis de conglomerados. Estos no indican el grado de correlación, pero permiten visualizar las relaciones de agrupación entre las categorías de acuerdo al grado de similitud de los datos codificados y las características compartidas.

El primer grupo de similitudes aparece en los temas referidos a flexibilidad (sistema de cuidados), comunicación, expresión y manejo emocional (sistema de conductas). Las madres perciben una relación entre las alteraciones en el desarrollo del lenguaje de los niños y la expresión emocional de estos: a menores habilidades comunicativas, mayores manifestaciones emocionales que ellas asocian con frustración o enojo (principalmente "rabietas"). Por esto, consideran que deben ser flexibles en su respuesta a estas expresiones y ensayar distintas conductas que resulten eficaces.

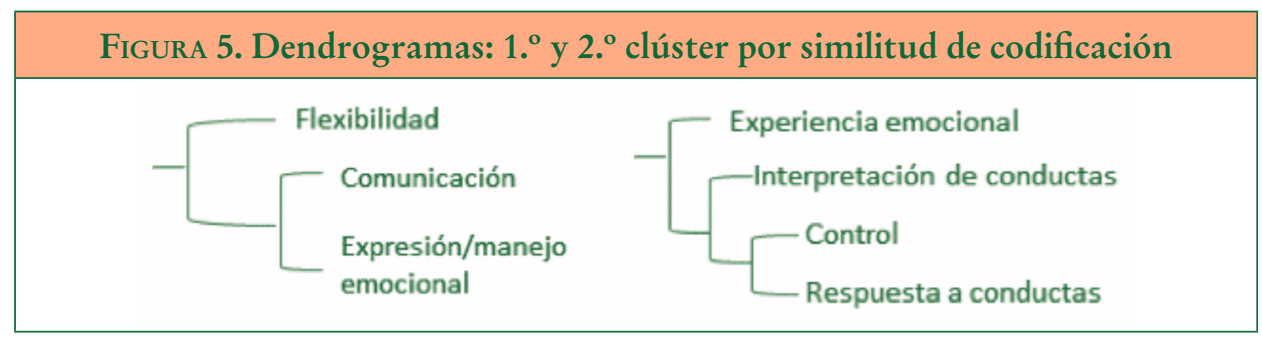

El segundo grupo alude a la experiencia emocional materna y su influencia en la capacidad para interpretar y responder a las conductas de sus hijos. En general, existe la percepción de que les cuesta comprender el comportamiento de los niños y asocian sus propios estados emocionales negativos (tristeza, frustración, confusión, culpa) con menor capacidad de responder favorablemente.

Hasta que no asumes ciertas cosas, creo que la capacidad de ayuda está mermada. [...] En los "días negros" que me frustro o me gana la incertidumbre, ayudo poco a mi hija. Me doy cuenta, soy consciente y esos días he comprobado que es mejor no trabajar en cosas con ella. Eso me ayuda a volcarme en lo que necesita y no en lo que yo quiero (A., 40 años). 


\section{EXPERIENCIAS DE APEGO EN MADRES DE NIÑOS CON RETRASO MADURATIVO: ANÁLISIS DE CONTENIDO \\ MARITZA GARCÍA TORO Y M. ${ }^{a}$ CRUZ SÁNCHEZ GÓMEZ}

A este respecto, sobresalen referencias al control de la conducta (enseñar límites y poner normas) como uno de los aspectos de la interacción materno-filial que genera dificultad.

La siguiente agrupación muestra dos clasificaciones: por un lado, la vacilación de las madres entre su función protectora y el fomento de la autonomía cuando el niño inicia las conductas de exploración; y, por otro lado, las conductas de los niños hacia la figura de apego (contacto visual, contacto físico y actitud del niño tras la separación).

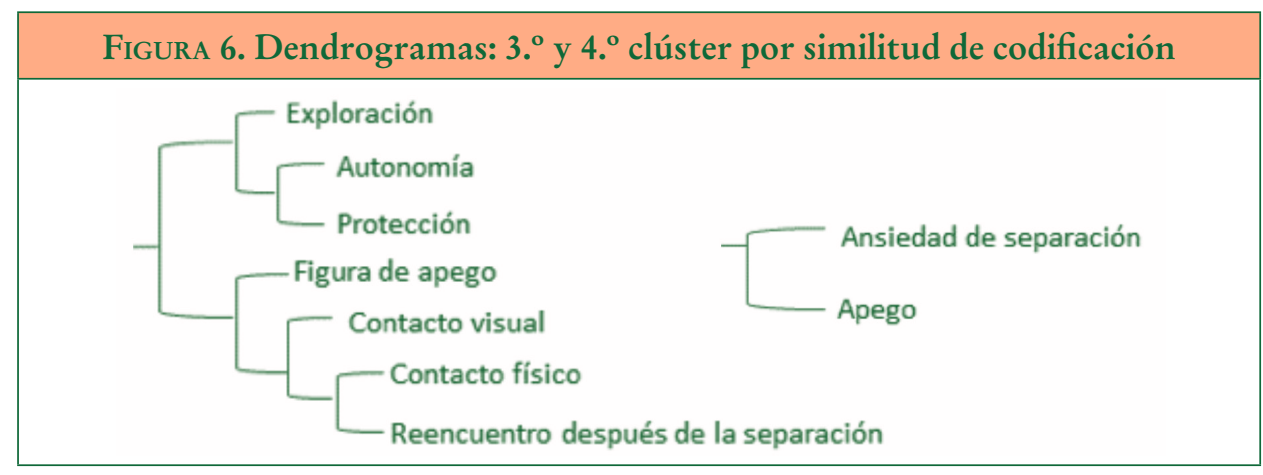

En la línea del anterior, el siguiente clúster muestra una asociación entre vínculo de apego y ansiedad de separación, pues es en la actitud del niño en esta situación (y en el reencuentro) donde las madres evalúan si su hijo tiene desarrollado el apego.

La próxima agrupación nos evidencia la relación entre el proceso de aceptación (nombrado como duelo) y el acompañamiento profesional. De otra parte, las narraciones apuntan a la incondicionalidad de las madres y la búsqueda de intimidad afectiva independientemente de las conductas de apego del niño. En lo relativo a los procesos de socialización y el juego en los niños, varían las experiencias: algunos niños muestran intencionalidad en la interacción con pares, pero no cuentan con habilidades de socialización y otros parecen no mostrar mayor interés.

\section{FiguRA 7. Dendrogramas: $5 .^{\circ}, 60^{\circ}$ y $7 .^{\circ}$ clúster por similitud de codificación}

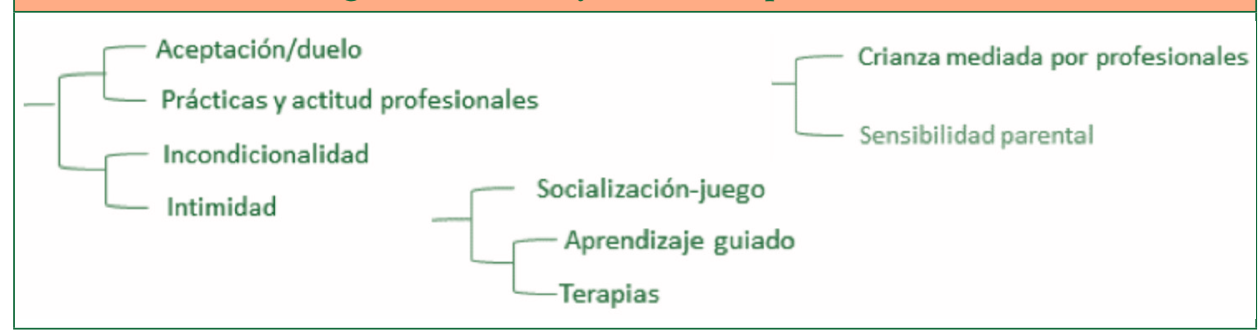

Otro ámbito en el que las madres muestran mucho interés y preocupación es en el desarrollo de habilidades y hay alusiones a la necesidad de guiar el aprendizaje, ya 
que este no siempre se da de manera espontánea. Aquí, las terapias juegan un papel importante y las madres reconocen que en algún momento abarca gran parte de la interacción parental. Sin embargo, también narran que esta tendencia disminuye con el paso del tiempo y con la aceptación de la situación del niño, de modo que se permiten mayores momentos de interacción desde el disfrute y la intimidad afectiva.

El último clúster apunta a la solicitud de intervención profesional por parte de las madres para recibir orientación sobre cómo atender las necesidades de sus hijos, lo cual parte del reconocimiento de que necesitan ayuda en el proceso de crianza.

Empiezas por asumir que tienes dificultades, luego, que necesitas ayuda, después por asumir que, aunque con ayuda, no vas a alcanzar los objetivos en poco tiempo, después por asumir que hay una incertidumbre inherente al proceso... (A., 40 años).

Para terminar, la Figura 8 representa gráficamente las correlaciones más fuertes entre las categorías de acuerdo con las coincidencias en la codificación, es decir, cuando en un fragmento de discurso se alude a una categoría también se alude a otra. Esto se corroboró con correlaciones de Pearson (Tabla 2).

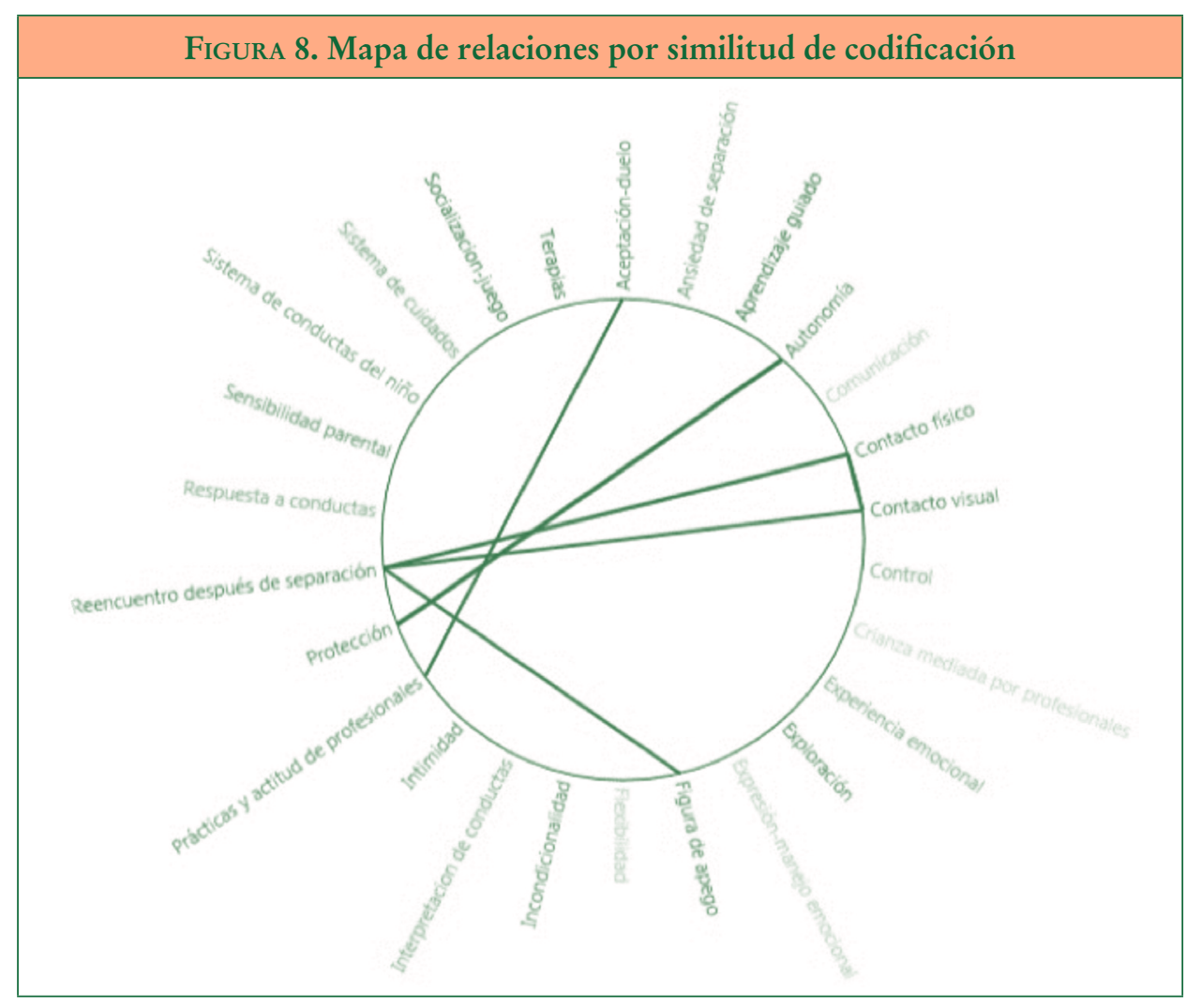

Ediciones Universidad de Salamanca / CC BY-NC-ND

Siglo Cero, vol. 51 (1), 2020, enero-marzo, pp. 89-109 


\section{EXPERIENCIAS DE APEGO EN MADRES DE NIÑOS CON RETRASO MADURATIVO: ANÁLISIS DE CONTENIDO \\ MARITZA GARCÍA TORO Y M. ${ }^{a}$ CRUZ SÁNCHEZ GÓMEZ}

Estas similitudes refuerzan lo mencionado anteriormente con relación a las conductas de apego que las madres ponen de relieve durante el reencuentro (contacto físico y contacto visual), que les permite evaluar el apego de sus hijos. También tiene una alta resonancia su función protectora que entra en conflicto con propiciar autonomía, así como las prácticas y actitud de los profesionales frente al proceso de aceptación, pues narran que las intervenciones se enfocan en la adquisición de habilidades y menos en el componente emocional, sobre todo de la familia.

\begin{tabular}{|c|c|}
\hline NODOS & COEFICIENTE DE PEARSON \\
\hline Protección $\backslash$ Autonomía & 1 \\
\hline Reencuentro $\backslash$ Contacto físico & 0,866 \\
\hline Contacto visual $\backslash$ Contacto físico & 0,84 \\
\hline Reencuentro\Figura de apego & 0,733 \\
\hline Reencuentro $\backslash$ Contacto visual & 0,727 \\
\hline Profesionales $\backslash$ Aceptación-duelo & 0,724 \\
\hline Exploración \Autonomía & 0,688 \\
\hline Protección $\backslash$ Exploración & 0,688 \\
\hline Terapias $\backslash$ Aprendizaje guiado & 0,666 \\
\hline
\end{tabular}

Del mismo modo, las madres expresan que, durante el proceso de evaluación, surgen diagnósticos provisionales que muchas veces son descartados y crean la sensación de "estar elaborando varios duelos", lo cual genera un desgaste emocional. Además, manifiestan que reciben información contradictoria o incompleta, pues "ni los mismos profesionales" tienen claro el origen de las dificultades de los niños y esto les ocasiona confusión y frustración. Por el contrario, los profesionales que brindan información clara, contención emocional, o, por lo menos, muestran disponibilidad para escuchar y aclarar dudas, permiten a las familias mayor tranquilidad y proactividad en las demandas diarias que implican la crianza con un hijo con retraso madurativo.

Estaba tan agobiada que no disfrutaba de mis hijos [...] No me relajaba, todo lo analizaba, y me sentía totalmente incomprendida [...] Todo mejoró al encontrar un cole donde le entienden y se adaptan a él y una terapeuta que trabaja día a día para que siga avanzando. Me transmiten tanta tranquilidad que yo he conseguido relajarme. Sé que es un camino largo y duro, con muchas curvas y cuestas, pero hay que recorrerlo con esperanza (A.S., 41 años).

\section{Discusión y conclusiones}

Este estudio se propuso perfilar experiencias de apego de un grupo de madres de niños con retraso madurativo a través del análisis de contenido de un foro virtual. En las narrativas se identificaron tres grandes categorías que corresponden a la literatura 
científica y una categoría emergente: sistema de cuidados, sistema de conductas y crianza mediada por profesionales.

El análisis revela que las madres experimentan reacciones emocionales intensas relacionadas con el diagnóstico, que comparan con el duelo y, desde su perspectiva, atraviesan las mismas fases de este proceso (negación, enfado, búsqueda de soluciones, dolor emocional, aceptación). A través de sus relatos se visualiza que esto permea gran parte de la experiencia emocional materna y es claro su impacto sobre el sistema de cuidados. Los resultados de este estudio apoyarían el trabajo de Feniger-Schaal y Oppenheim (2013), quienes señalan que a las familias les cuesta avanzar en el proceso de aceptación y reajustar la representación de su hijo cuando el diagnóstico no es percibido como permanente. En tal caso, la terminología, la actitud y las prácticas de los profesionales podrían tener incidencia, pues las madres manifiestan que, si bien comprenden las razones de esta categoría provisional (retraso madurativo) y la importancia de no "etiquetar" a los niños cuando no hay claridad diagnóstica, también reconocen que les cuesta asumir algo cuando no tienen certeza de qué se trata. Además, el desgaste emocional asociado a lo que denominan elaborar "varios duelos" tiene implicaciones en la disponibilidad materna.

En las primeras etapas, las madres están enfocadas en la clarificación del diagnóstico, en las terapias que requiere su hijo y en el desarrollo de habilidades, lo cual disminuye los espacios de interacción con su hijo centrados en el placer y el disfrute. Estos primeros momentos se caracterizan por una actitud de observación y análisis de los hijos para detectar signos de alarma, mejorías, dificultades o encontrar sentido a sus comportamientos, es decir, la representación mental del hijo gira en torno al diagnóstico. Posteriormente, algunas madres avanzan hasta la aceptación y de allí a la valoración, reconociendo cualidades y esfuerzos del niño, sin desconocer las limitaciones, lo que permite mayor intimidad afectiva, interacciones menos estructuradas y menos centradas en fines terapéuticos.

Por otro lado, Ainsworth et al. (1978) plantean que la sensibilidad materna comienza con notar los signos del niño, por ejemplo, sus señales de dolor, cansancio, enfermedad, disfrute, entre otros, y a partir de allí el cuidador determina las necesidades del infante y responde de acuerdo a esta interpretación. Las participantes del foro se muestran atentas a las señales de sus hijos (en ocasiones, hipervigilantes) y disponibles, pero les cuesta el proceso de interpretación, y no tienen claro si responden apropiadamente; destacan que en este proceso influyen tanto sus estados emocionales como la impredictibilidad de la conducta de los niños. Así mismo, la ausencia de conductas de apego genera frustración en las madres, pues involucra lo más primordial de la experiencia materna que es establecer un vínculo con su hijo. Sin embargo, se observa que las emociones y sentimientos positivos, como la esperanza o la alegría y el deseo de lograr intimidad afectiva con el niño, les permiten ser más flexibles, buscar distintas alternativas y estrategias para vincularse. Esto coincide con Boström, Broberg y Hwang (2009), quienes sugieren que las emociones y pensamientos positivos de las figuras parentales hacia el niño pueden ser un factor protector que promueva la habilidad para comprender las necesidades de los hijos. En cuanto a la función materna, los ámbitos que se ponen de relieve son la promoción de autonomía vs. protección, la transmisión de normas y de límites (control). 
Las investigaciones han subrayado la asociación entre sensibilidad parental y seguridad del apego (Lucassen et al., 2011; van Ijzendoorn et al., 2007) y entre resolución del diagnóstico y sensibilidad parental (Feniger-Schaal y Oppenheim, 2013; Oppenheim et al. 2007), de modo que es importante continuar profundizando en lo encontrado en la presente investigación sobre la relación entre acompañamiento profesional y resolución del diagnóstico, con el fin de que los profesionales puedan hacer modificaciones a sus prácticas y actitudes para brindar una atención en favor del bienestar emocional parental (Floyd y Dago, 2014; Martínez y Calet, 2015), y para la construcción de vínculos de apego más favorables. Para lograr lo anterior, se requiere contar con las experiencias de familias en otros contextos y, teniendo en cuenta que actualmente hay una tendencia hacia una paternidad más activa, es necesario contemplar los discursos de los padres en futuras investigaciones sobre el tema.

Por último, se ha establecido que el apego seguro puede ser un factor protector para prevenir problemas emocionales en niños con alteraciones del desarrollo, pues favorece la regulación emocional en situaciones estresantes y el niño emplea a las figuras de apego para resolver la situación que causa el estrés si no cuenta con recursos emocionales (Janssen et al., 2002), así que los cuidadores deben contar con herramientas para promover la seguridad en el apego y mejorar su bienestar socioafectivo (Baker, Fenning, Crnic, Baker y Blacher, 2007). Los resultados obtenidos en este estudio acentúan la importancia de implementar acompañamiento emocional a las familias durante todo el proceso diagnóstico para sobrellevar los sentimientos negativos asociados y ofrecer citas de seguimiento para continuar discutiendo el diagnóstico y los momentos transicionales a lo largo del desarrollo del niño (Fletcher, 2016). Así mismo, prestar atención a la terminología empleada por los profesionales e incluir intervenciones orientadas no solo a la adquisición de destrezas en los niños, sino a la estimulación de la disponibilidad parental (Morin, 2012; Moss, Dubois-Comtois y Cyr, 2010), la aceptación incondicional y la valoración, la adaptación a las capacidades del niño y la flexibilidad ante sus comportamientos, al igual que una estimulación variada, que esté ligada a la interacción emocional íntima.

En este estudio se advierte la existencia de varios sesgos: en primer lugar, es posible que las familias que acuden a foros de discusión sean aquellas que se sienten más desorientadas con respecto a la situación de sus hijos y su rol parental. En segundo lugar, parece existir "contaminación" del discurso de las madres por la exposición a internet y al discurso de profesionales, tal como revela la terminología usada al describir el proceso de "duelo", las terapias y los criterios diagnósticos. Por otro lado, la muestra estaba compuesta solo por el relato de mujeres, debido a la baja presencia masculina en el foro y a que su participación en temas relacionados con la dimensión emocional es escasa.

\subsection{Consideraciones éticas}

Aunque se asume que la investigación realizada en espacios públicos (lo que es extensible a los medios de comunicación social: prensa, radio, televisión o internet) 


\section{EXPERIENCIAS DE APEGO EN MADRES DE NIÑOS CON RETRASO MADURATIVO: ANÁLISIS DE CONTENIDO \\ MARITZA GARCÍA TORO Y M. ${ }^{a}$ CRUZ SÁNCHEZ GÓMEZ}

no exige consentimiento informado, pues se da por sentado que las personas saben que su comunicación en estos espacios es abierta y está expuesta a ser observada, esto no exime de consideraciones éticas (Estalella y Ardèvol, 2007). En el presente trabajo se siguieron los principios de la investigación con personas: respeto a la dignidad, la seguridad y la privacidad (Sociedad Interamericana de Psicología, 2008). Los nombres de usuario de las madres se redujeron a iniciales y se suprimieron los nombres de los niños, así como cualquier dato que revele su identidad.

\section{Referencias bibliográficas}

Ainsworth, M., Blehar, M. C., Waters, E. y Wall, S. (1978). Patterns of attachment. Hillsdale, NJ: Lawrence Erlbaum.

Alexander, S. L., Frederico, M. y Long, M. (2018). Attachment and children with disabilities: knowledge and views of early intervention professionals. Children Australia, 43(4), 245-254. https://doi.org/10.1017/cha.2018.38.

Arriazu, R. (2007). ¿Nuevos medios o nuevas formas de indagación? Una propuesta metodológica para la investigación social on-line a través del foro de discusión. Forum Qualitative Sozialforschung/Forum: Qualitative Social Research, 8(3). https://doi.org/10.17169/ fqs-8.3.275.

Baker, J. K., Fenning, R. M., Crnic, K. A., Baker, B. L. y Blacher, J. (2007). Prediction of social skills in 6-years-old children with and without developmental delays: contributions of early regulation and maternal scaffolding. American Journal on Mental Retardation, 112, 375-391.

Boström, P. K., Broberg, M. y Hwang, P. (2009). Parents' descriptions and experiences of young children recently diagnosed with intellectual disability. Child: Care, Health and Development, 36(1), 93-100. https://doi.org/10.1111/j.1365-2214.2009.01036.x.

Bowlby, J. (1969). Attachment. Attachment and loss (vol. I). Londres: Hogarth Press.

Bowlby, J. (1973). Anxiety y anger. Attachment and loss (vol. II). London: Hogarth Press.

Bowlby, J. (1980). Sadness y depression. Attachment and loss (vol. III). London: Hogarth Press.

Campos-Castelló, J. (2013). Retraso madurativo neurológico. Revista de Neurología, 57(supl. 1), 211-219.

Chandramuki, Dr., Venkatakrishnashastry, I. y Vranda, M. (2012). Attitudes of Parents towards Children with specific learning disabilities. Disability, CBR and Inclusive Development, 13(1), 63-69. https://doi.org/10.5463/DCID.v23i1.47.

Creswell, J. W. (2013). Research design: qualitative, quantitative, and mixed methods approaches (3. ${ }^{\mathrm{a}}$ ed.). Thousand Oaks, CA, EE. UU.: SAGE.

Czornyj, L. (2004). Retraso madurativo. Aspectos neurológicos. En H. Lejarraga, El desarrollo del niño en contexto (pp. 193-215). Buenos Aires: Paidós.

Delgado, C. (2014). Viajando a Ítaca por los mares cuantitativos, manual de ruta para investigar en grado y en postgrado. Salamanca: Amaru.

Dyches, T. T., Smith, T. B., Korth, B. B., Roper, S. O. y Mandleco, B. (2012). Positive parenting of children with developmental disabilities: a meta-analysis. Research in Developmental Disabilities, 33, 2213-2220. https://doi.org/10.1016/j.ridd.2012.06.015.

Estalella, A. y Ardèvol, E. (2007). Ética de campo: hacia una ética situada para la investigación etnográfica de internet. Forum Qualitative Sozialforschung/Forum: Qualitative Social Research, 8(3). 


\section{EXPERIENCIAS DE APEGO EN MADRES DE NIÑOS CON RETRASO MADURATIVO: ANÁLISIS DE CONTENIDO \\ MARITZA GARCÍA TORO Y M. ${ }^{a}$ CRUZ SÁNCHEZ GÓMEZ}

Feniger-Schaal, R. y Oppenheim, D. (2013). Resolution of the diagnosis and maternal sensitivity among mothers of children with intellectual disability. Research in Developmental Disabilities, 34, 306-313. https://doi.org/10.1016/j.ridd.2012.08.007.

Fiamenghi, G. A., Vedovato, A. G., Meirelles, M. C. y Shimoda, M. E. (2010). Mothers' interaction with their disabled infants: two case studies. Journal of Reproductive and Infant Psychology, 28(2), 191-199. https://doi.org/10.1080/02646830903295042.

Fletcher, H. (2016). Attachment relashionships beetwin parents and their children: the impact of "the loss of the healthy child". En H. Fletcher, A. Flood y D. J. Hare (Eds.), Attachment in intellectual and developmental disability: a clinical guide to practices and research. John Wiley and Sons: Londres.

Floyd, M. y Dago, R. (2014). La importancia de buena comunicación entre el médico y el niño, la familia, y otros profesionales. Revista Nacional e Internacional de Educación Inclusiva, 7, 148-163.

Hernández, R., Fernández, C. y Baptista, P. (2010). Metodología de la investigación (6. ${ }^{2}$ ed.). D. F., México: McGraw Hill.

Howe, D. (2006). Disabled children, parent-child interaction and attachment. Child and Family Social Work, 11, 95-106. https://doi.org/10.1111/j.1365-2206.2006.00397.x.

Hsien, R. L., Hsieh, W. H. y LeE, W. C. (2016). Short-term family-centered workshop for children with developmental delays enhances family functioning and satisfaction: a prospective clinical trial. Medicine, 95(31), e4200. https://doi.org/10.1097/MD.0000000000004200.

Janssen, C., Schuengel, C. y Stolk, J. (2002). Understanding challenging behaviour in people with severe and profound intellectual disability: a stress-attachment model. Journal of Intellectual Disability Research, 46(6), 445-453.

LaMont, M. (2011). Mother-child attachment and preschool behavior problems in children with developmental delay. Dissertation Abstracts International, 72, 1827.

Lewallen, A. C. y Neece, C. L. (2015). Improved social skills in children with developmental delays after parent participation in MBSR: The role of parent-child relational factors. Journal of Child and Family Studies, 24(10), 3117-3129. https://doi.org/10.1007/s10826015-0116-8.

López, F. y Ortiz, M. J. (2001). El desarrollo del apego en la primera infancia. En F. López, I. Etxebarria, M. J. Fuentes y M. J. Ortiz (Coords.), Desarrollo afectivo y social (pp. 41-64). Madrid: Pirámide.

Lucassen, N., Tharner, A., van Ijzendoorn, M. H., Bakermans-Kranenburg, M. J., Volling, B. L., Verhulst, F. C., Lambregtse-van den Berg, M. P. y Tiemeier, H. (2011). The association between paternal sensitivity and infant-father attachment security: a metaanalysis of three decades of research. Journal of Family Psychology, 25(6), 986-992. https:// doi.org/10.1037/a0025855.

Martínez, A. y Calet, N. (2015). Intervención en Atención Temprana: Enfoque desde el ámbito familiar. Escritos de Psicología, 8(2), 33-42. Recuperado de http://scielo.isciii.es/scielo. php?script=sci_arttext\&amp;pid=S1989-38092015000200006.

Morin, I. (2012). Les troubles de l'attachement en déficience intellectuelle. Québec: SQETGC.

Moss, E., Dubois-Coмтоis, K. у Cyr, C. (2010). Efficacy of a home-visiting intervention aimed at improving maternal sensitivity, child attachment, and behavioral outcomes for maltreated children: a randomized control trial. Dev Psychopathol, 195-210. https://doi. org/10.1017/S0954579410000738.

Narbona, J. y Schlumberger, É. (2008). Retraso Psicomotor. En J. Narbona García y C. Casas Fernández, Protocolos de Neurología (pp. 151-157). Madrid: SENP-AEP. 


\section{EXPERIENCIAS DE APEGO EN MADRES DE NIÑOS CON RETRASO MADURATIVO: ANÁLISIS DE CONTENIDO \\ MARITZA GARCÍA TORO Y M. ${ }^{a}$ CRUZ SÁNCHEZ GÓMEZ}

Oppenheim, D., Dolev, S., Koren-Karie, N., Sher-Censor, E., Yirmiya, N. y Salomon, S. (2007). Mother's reactions to their child's diagnosis: relations with security of attachment. En D. Oppenheim y D. F. Goldsmith, Attachment theory in clinical work with children bridging the gap between research and practice (pp. 109-136). New York, NY, US: Guilford Press.

Pérez-Salas, C. P. y Santelices, M. P. (2009). Vinculación en niños con discapacidad intelectual: Reflexiones para la promoción de un apego seguro. Revista de Psicopatología y Salud Mental del Niño y del Adolescente, 14, 17-25.

Rodríguez, G. y Gómez, M. (2010). Análisis de contenidos y textual de datos cualitativos. En S. Nieto Martín (Coord.), Principios, métodos y técnicas esenciales para la investigación educativa (pp. 191-219). Madrid: Dykinson.

Rutgers, A. H., van Ijzendoorn, M. H., Bakermans-Kranenburg, M. J., Swinkels, S. H. N., van Daalen, E., Dietz, C., Naber, F. B. A., Bui Telaar, J. y van Engeland, H. (2007). Autism, attachment and parenting: a comparison of children with autism spectrum disorder, mental retardation, language disorder, and non-clinical children. Journal of Abnormal Psychology, 35, 859-870. https://doi.org/10.1007/s10802-007-9139-y.

Sánchez, M. C., Delgado, M. C. y Santos, M. C. (2012). El proceso de investigación cualitativa. Manual de procedimiento: ejemplificación con una tesis doctoral. Valladolid: Edintras.

Schuengel, C., Clasien de Schipper, J., Sterkenburg, P. y Kef, S. (2013). Attachment, Intellectual Disabilities and Mental Health: Research, Assessment and Intervention. Journal of Applied Research in Intellectual Disabilities, 26, 34-46. https://doi.org/10.1111/jar.12010.

Schuengel, C., Kef, S., Damen, S. y Worm, M. (2010). 'People who need people': attachment and professional caregiving. Journal of Intellectual Disability Research, 54(supl. 1), 38-47. https://doi.org/10.1111/j.1365-2788.2009.01236.x.

Sociedad Interamericana de Psicología (26 de julio de 2008). Declaración Universal de Principios Éticos para Psicólogas y Psicólogos. Recuparado de Biblioteca Virtual en Psicología, Universidad de Buenos Aires: http://bvs.psi.uba.ar/local/File/DeclaracionPrinEticosSIP.PDF.

Teague, S. J., Newman, L. K., Tonge, B. J. y Gray, K. M. (2018). Caregiver mental health, parenting practices, and perceptions of child attachment in children with autism spectrum disorder. Journal of Autism and Developmental Disorders, 48(8), 2642-2652. https://doi. org/10.1007/s10803-018-3517-x.

TóJAR, J. C. (2006). Investigación cualitativa: comprender y actuar. Madrid: La Muralla.

Van Ijzendoorn, M., Rutgers, A., Bakermans-Kranenburg, M., van Daalen, E., Dietz, C., Swinkels, S., van Daalen, E., Dietz, C., Naber, F., Buitelaar, J. K. y van EngeLAND, H. (2007). Parental sensitivity and attachment in children with autism spectrum disorder: comparison with children with mental retardation, with language delays, and with typical development. Child Development, 78(2), 597-608. https://doi.org/10.1111/j.14678624.2007.01016.x. 


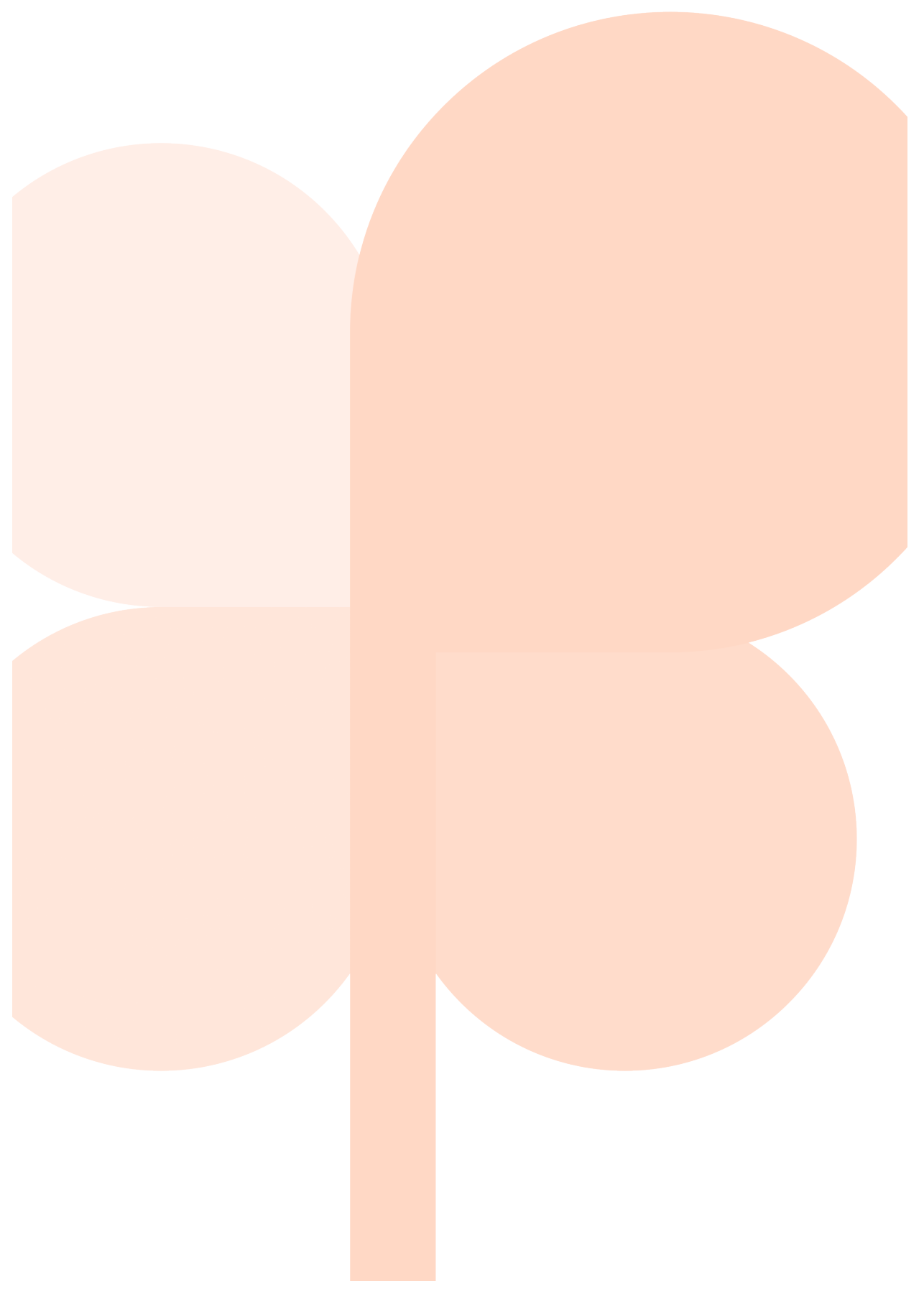

